\title{
Caracterização Nutricional de Pacientes com Úlceras Crônicas de Membros Inferiores em Tratamento no Ambulatório de Feridas do Campus Cedeteg da UNICENTRO, Guarapuava-PR
}

\section{Nutritional Characterization of Patients with Chronic Ulcer of Lower Limb in the Outpatient Treatment of Wounds of the Campus Cedeteg Unicentro, Guarapuava-PR}

\author{
Michele Dal Santos ${ }^{\mathrm{a}}$; Silvana Franco ${ }^{\mathrm{b}}$; Fabiane La Flor Ziegler Sanches ${ }^{\mathrm{c} *}$; Raquel Rosalva ${ }^{\mathrm{b}}$; Jaqueline Hack \\ Nathália Meottia; Marcela Magro ${ }^{a}$
}

${ }^{a}$ Universidade Estadual do Centro-Oeste, Curso de Nutrição, PR, Brasil

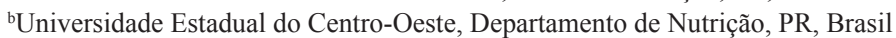

'Universidade Federal de Mato Grosso do Sul, Curso de Nutrição, MS, Brasil

*E-mail: fabianelaflor@gmail.com

Recebido: 28 de fevereiro de 2014; Aceito: 23 de junho de 2014

\begin{abstract}
Resumo
Pacientes com úlceras crônicas, muitas vezes, apresentam hábitos alimentares inadequados que dificultam a cicatrização, seja pela falta de nutrientes ou pelo excesso destes. Sendo assim, objetivou-se caracterizar o estado nutricional e a prevalência de doenças crônicas de pacientes com úlceras nos membros inferiores em tratamento no Ambulatório de Feridas do Campus Cedeteg da Unicentro, Guarapuava - PR. Estudo transversal quantitativo, constituído por 36 pacientes. Os dados foram obtidos em formulários estruturados. Para análise estatística foi utilizado o programa SPSS versão 15.0 para Windows, considerando-se 5\% de significância. As idades variaram de 40 a 76 anos. O tipo de úlcera mais presente na amostra foi a venosa com $72 \%(\mathrm{n}=26)$, seguido de neuropática por hanseníase com $20 \%(\mathrm{n}=7)$. A doença crônica mais prevalente foi hipertensão arterial sistêmica $(52,8 \%)$, seguido de insuficiência venosa $(30,6 \%)$, diabetes melittus $(19,4 \%)$ e obesidade $(33,3 \%)$. Segundo o Índice de Massa Corporal (IMC), 41,6\% apresentou eutrofia e de acordo com a circunferência da cintura (CC), 75\% da amostra apresentou a medida acima do recomendado. Os valores de ingestão de nutrientes encontraram-se abaixo do recomendado para homens e para mulheres para vitamina $A(p=0,0001$ e $p=0,002)$, vitamina $C(p=0,001$ e 0,016$)$, zinco $(p=0,080$ e $p=0,001)$ e de proteínas somente para as mulheres $(\mathrm{p}=0,020)$. Ainda, o grupo dos homens, apresentou uma ingestão adequada de calorias e elevada de proteínas e ferro. Percebeu-se que esses pacientes necessitam de estratégias voltadas para a alimentação e controle das comorbidades, a fim de favorecer a cicatrização das úlceras.
\end{abstract}

Palavras-chave: Úlcera da Perna. Estado Nutricional. Doença Crônica. Cicatrização.

\begin{abstract}
Patients with chronic ulcers often have poor dietary habits that hinder the healing process, or by the lack of nutrients or excess thereof. Therefore, the objective was to characterize the nutritional status and the prevalence of chronic diseases of patients with leg ulcers treated at the Clinic Wound Campus CEDETEG of Unicentro, Guarapuava - PR. Quantitative cross-sectional study consisting of 36 patients. Data were obtained from structured forms. Statistical analysis was performed using the SPSS version 15.0 for Windows, considering $5 \%$ significance level. Their ages ranged from 40 to 76 years. The type of ulcer longer present in the venous sample was $72 \%(n=26)$, followed by neuropathic leprosy with $20 \%(n=7)$. The disease was most prevalent chronic hypertension (52.8\%), followed by venous insufficiency (30.6\%), diabetes mellitus (19.4\%) and obesity (33.3\%). According to the body mass index (BMI), 41.6\% had normal weight and according to waist circumference (WC), $75 \%$ showed the measure above recommended. The values of nutrient intake were found below recommended for men and women for vitamin $A(p=0.000$ and $p=0.002)$, vitamin $C(p=0.00$ and 0.016$)$, zinc $(p=0.080$ and $p=0.001)$ and protein only for women $(p=0.020)$. Still, the group of men had an adequate intake of calories and high in protein and iron. It was noticed that these patients need strategies for power and control of comorbidities in order to promote the healing of ulcers.
\end{abstract}

Keywords: Leg Ulcer. Nutritional Status. Chronic Disease. Wound Healing.

\section{Introdução}

A úlcera de perna é definida como uma ulceração abaixo do joelho em qualquer parte da perna ou pé, causando destruição de estruturas cutâneas, tais como epiderme e derme, podendo afetar, também, tecidos mais profundos ${ }^{1,2}$. Pode ser classificada como uma ferida crônica quando não cicatriza por um período de seis semanas ou mais ${ }^{3}$.

$\mathrm{O}$ aparecimento das úlceras crônicas de membros inferiores pode estar associado a várias causas, tais como: doença venosa crônica, doença arterial periférica, neuropatias, hipertensão arterial sistêmica - HAS, trauma físico, anemia falciforme, infecções cutâneas, doenças inflamatórias, neoplasias e alterações nutricionais como hipoproteinemia, diminuição sérica de vitamina $\mathrm{A}$, vitamina $\mathrm{C}$, zinco e ferro ${ }^{4-6}$.

Os tipos mais comuns de úlceras de membros inferiores, de acordo com sua etiologia, são as de origem venosa, abrangendo $70 \%$ dos casos. Estes pacientes costumam apresentar insuficiência venosa crônica - IVC ou ter história anterior de trombose venosa profunda - TVP. Na sequência encontram-se as úlceras de origem arterial, com ocorrência de 10 a $20 \%$, comumente causadas por aterosclerose ou por outras doenças arteriais, como a tromboangeíte obliterante. As de etiologia mista (associações entre arteriais, venosas e neuropáticas) comportam de 10 a $15 \%$ dos $\operatorname{casos}^{7-10}$.

Úlceras neuropáticas são comuns em algumas patologias 
que acometem o sistema nervoso periférico, como a hanseníase, o alcoolismo e o diabetes mellitus - DM, doenças endêmicas no Brasil. Essas patologias podem afetar os nervos periféricos, causando danos às fibras autônomas, sensitivas e motoras $^{11}$.

As feridas crônicas interferem na produtividade e na qualidade de vida da população, sendo que, as mais encontradas nos serviços da rede básica de saúde brasileira são as úlceras venosas, as arteriais, as hipertensivas, as de pressão e as neuropáticas, geralmente apresentando cura demorada e resposta terapêutica variável ${ }^{11}$.

Diversos fatores podem afetar negativamente a cicatrização como a perfusão tecidual, infecção, corticosteroides, diabetes mellitus, quimioterapia, idade, fumo, entre outros ${ }^{12}$. No entanto, o processo de cicatrização de feridas também é influenciado significativamente pelo estado nutricional do portador da lesão, considerando-se que a reparação tecidual depende de um aporte calórico, de proteínas, carboidratos, lipídios, vitaminas e minerais ${ }^{13,14}$.

Analisando a importância de estudos sobre o tema, o objetivo do presente trabalho foi caracterizar o estado nutricional e a prevalência de doenças crônicas de pacientes com úlceras nos membros inferiores em tratamento no Ambulatório de Feridas do Campus Cedeteg da UNICENTRO, Guarapuava-PR.

\section{Material e Métodos}

\subsection{Desenho do estudo}

Trata-se de um estudo do tipo quantitativo transversal. A coleta foi realizada no período de março a maio de 2012, com 36 pacientes adultos e idosos que apresentavam úlceras de perna crônicas e estavam em tratamento no Ambulatório de Feridas do Campus Cedeteg da UNICENTRO, GuarapuavaPR.

A coleta de dados do presente trabalho se iniciou após a aprovação do projeto de pesquisa pelo Comitê de Ética em Pesquisa da Universidade Estadual do Centro-Oeste (COMEP/ UNICENTRO), através do Ofício nº 549/2011.

Os critérios de inclusão dos pacientes foram: estar presente no local durante o período de coleta de dados ter assinado o Termo de Consentimento Livre e Esclarecido TCLE, confirmação do diagnóstico de úlceras nos membros inferiores, e pertencer a faixa etária de adultos e idosos.

Foram excluídos os pacientes sem capacidade cognitiva para entender e responder os questionamentos e pacientes que apresentassem úlceras em outros locais, como na região das nádegas.

\subsection{Avaliação da prevalência de doenças crônicas}

A avaliação dos tipos de úlceras e das comorbidades foi realizada através do relato dos pacientes, diagnósticos fechados por médico e enfermeiros e observação de prontuários. Estes dados foram anotados em formulários elaborados para este fim.

\subsection{Avaliação antropométrica}

Foi realizada através da aferição do peso $(\mathrm{kg})$, estatura (m), pregas cutâneas $(\mathrm{mm})$ e circunferência da cintura $(\mathrm{cm})$. Foram seguidas as metodologias preconizadas pelo SISVAN (Sistema de Vigilância Alimentar e Nutricional) ${ }^{15}$.

A partir desses dados, foi calculado o Índice de Massa Corporal (IMC), utilizando os pontos de corte preconizados pela Organização Mundial da Saúde ${ }^{16}$ para adultos e da Organização Pan Americana de Saúde ${ }^{17}$ para idosos.

Para mensuração do peso foi utilizada balança digital portátil, com precisão de $100 \mathrm{~g}$ da marca Britânia. Para a obtenção da estatura foi usado estadiômetro compacto Wiso (0 a $200 \mathrm{~cm}$ ), porém para os indivíduos que necessitavam de estimação da estatura foi realizada a aferição da altura do joelho com uma fita métrica inelástica. $\mathrm{O}$ indivíduo deveria estar sentado ou em posição supina com joelho esquerdo flexionado em um ângulo de $90^{\circ}$. O comprimento entre o calcanhar e a superfície superior do joelho foi aferido e a altura foi posteriormente obtida por meio das equacões de estimativa de altura de Chumlea ${ }^{18}$, de acordo com cada gênero.

As pregas cutâneas aferidas foram: prega cutânea tricipital - PCT, prega cutânea bicipital - PCB, prega cutânea subescapular - PCSE e prega cutânea suprailíaca - PCSI através do uso de adipômetro da marca Cescorf ${ }^{\mathbb{B}}$. A circunferência muscular do braço - CMB foi obtida através dos valores da circunferência do braço - CB e a prega cutânea triciptal - PCT.

O percentual de gordura corporal $(\% \mathrm{GC})$ foi obtido pela soma das pregas cutâneas, empregando o padrão de referência preconizado nas tabelas de Pollock e Wilmore ${ }^{19}$. O risco de doenças associadas ao excesso de peso foi determinado pela circunferência da cintura - CC, utilizando o preconizado pela Organização Mundial da Saúde - $\mathrm{OMS}^{16}$ que estabelece como ponto de corte para risco aumentado a medida de CC igual ou superior a $94 \mathrm{~cm}$ em homens e igual ou superior a $80 \mathrm{~cm} \mathrm{em}$ mulheres.

\subsection{Avaliação do consumo alimentar}

A avaliação da ingestão calórica e de nutrientes de todos os avaliados foi efetuada com a utilização de um inquérito alimentar do tipo Dia Alimentar Habitual - DHA, onde os participantes relataram o que consumiram habitualmente, além da quantidade, horário, número e tipo de refeições (desjejum, almoço, jantar e lanches), alimentos e preparações de cada refeição.

Os dados obtidos das medidas caseiras foram convertidos em gramas para o cálculo das calorias e nutrientes ingeridos, utilizando-se o Software AVANUTRI ${ }^{\circledR}$ versão 3.1.5. Com base na literatura de pesquisas com indivíduos portadores de úlceras crônicas, optou-se por avaliar a adequação da alimentação, comparando a ingestão energética, de proteínas, vitamina $\mathrm{A}$, vitamina $\mathrm{C}$, ferro e zinco e as respectivas necessidades e recomendações de cada indivíduo, segundo gênero e faixa etária. 
A necessidade energética foi calculada pela equação de Harris Benedict (HBE) com fator atividade 1,3 (estado ambulatorial) e fator lesão 1,0. Este último fator foi utilizado para diminuir o risco de superestimação do gasto energético total $^{20,21}$, conforme a metodologia empregada por Tobón ${ }^{6}$. A ingestão foi comparada com as recomendações de proteínas $(0,8 \mathrm{~g} / \mathrm{kg} / \mathrm{dia})$, vitaminas e minerais utilizaram-se as recomendações das Dietary Reference Intakes - DRIs para cada gênero e faixa etária ${ }^{22-24}$, considerando a porcentagem de adequação de $90 \%$ a $110 \%$.

\subsection{Análise estatística}

Os dados coletados foram tabulados em planilha Excel ${ }^{\circledR}$ 2007, representados através de tabelas, gráficos e pelos testes estatísticos, com auxílio do software SPSS versão 15.0 para Windows. As variáveis foram descritas em mediana e variância interquartilica, frequência relativa e absoluta, de acordo com a necessidade.

O teste de Shapiro-Wilk foi utilizado para determinar a distribuição dos dados. O teste pareado de Wilcoxon foi empregado para comparar a ingestão habitual de nutrientes às recomendações. O teste de Mann-Whitney foi utilizado na comparação intergênero para características antropométricas. O nível de significância adotado para todos os testes foi de $5 \%(\mathrm{p}<0,05)$.

\section{Resultados e Discussão}

O estudo foi composto por 36 pacientes, sendo que 18 (50\%) do gênero feminino e 18 (50\%) do masculino, com idade entre 40 e 76 anos. A amostra apresentou idade média de $60 \pm 10,9$ anos. Quanto à faixa etária dos pacientes $19(52,8 \%)$ eram adultos e 17 (47,2\%) eram idosos.

Como se pode observar no gráfico da Figura 1, a maior prevalência encontrada foi de úlceras do tipo venosa com $72 \%(n=26)$, seguido de neuropática por hanseníase com $20 \%$ $(n=7)$ e outros tipos com $8 \%(n=3)$.

Figura 1: Prevalência dos tipos de úlceras nos pacientes atendidos no Ambulatório de Feridas do Campus Cedeteg, Unicentro, Guarapuava - PR.

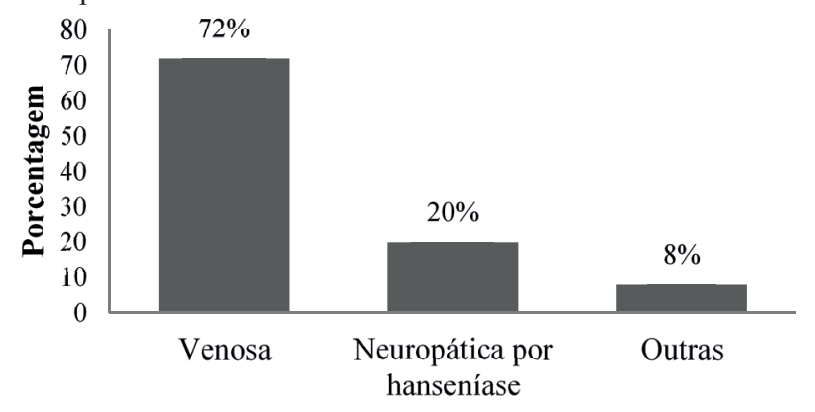

Foram investigadas doenças crônicas frequentemente associadas às úlceras de perna, como hipertensão arterial sistêmica (presente em 52,8\%, n=19), insuficiência venosa
(30,6\%, $\mathrm{n}=11)$, diabetes mellitus $(19,4 \%, \mathrm{n}=7)$ e obesidade $(33,3 \%, n=12)$. Pacientes que apresentaram nenhuma doença associada às úlceras corresponderam a 27,8\% $(n=10)$ da amostra (Figura 2).

Figura 2: Prevalência de doenças crônicas associadas às úlceras nos pacientes atendidos no Ambulatório de Feridas do Campus Cedeteg, Unicentro, Guarapuava -PR.

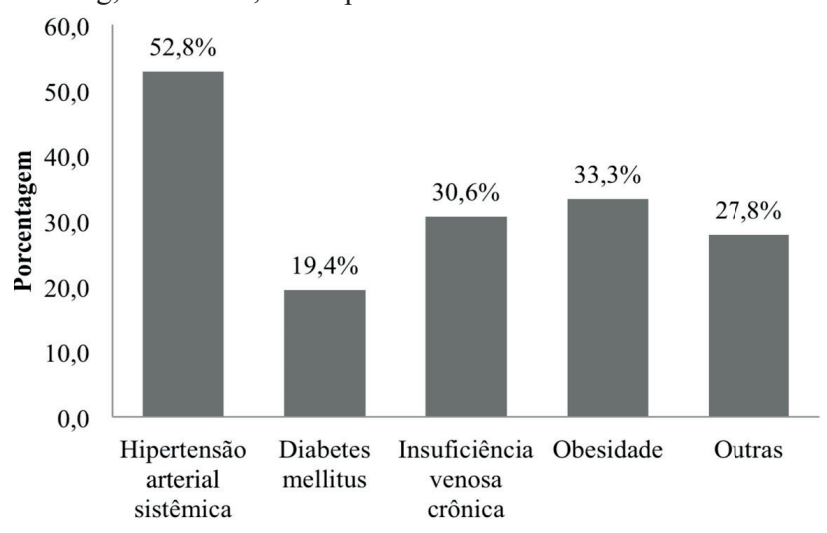

Os dados referentes ao índice de massa corporal - IMC e a circunferência da cintura - CC podem ser observados na Tabela 1.

Tabela 1: Classificação do estado nutricional dos pacientes segundo IMC e CC de pacientes atendidos no Ambulatório de Feridas do Campus Cedeteg, Unicentro, Guarapuava -PR.

\begin{tabular}{clcccccc}
\hline & & \multicolumn{2}{c}{$\begin{array}{c}\text { Homens } \\
(\mathbf{n}=\mathbf{1 8})\end{array}$} & \multicolumn{2}{c}{$\begin{array}{c}\text { Mulheres } \\
(\mathbf{n = 1 8})\end{array}$} & \multicolumn{2}{c}{$\begin{array}{c}\text { Total } \\
(\mathbf{n}=\mathbf{3 6})\end{array}$} \\
\cline { 3 - 8 } & $\mathbf{n}$ & $\mathbf{\%}$ & $\mathbf{n}$ & $\mathbf{\%}$ & $\mathbf{n}$ & $\mathbf{\%}$ \\
\hline \multirow{3}{*}{ IMC } & Eutrofia & 9 & 50,0 & 6 & 33,3 & 15 & 41,6 \\
& Sobrepeso & 3 & 16,6 & 6 & 33,3 & 9 & 25 \\
& Obesidade & 6 & 33,3 & 6 & 33,3 & 12 & 33,3 \\
\hline \multirow{2}{*}{ CC } & Sem risco* & 5 & 27,7 & 4 & 22,2 & 9 & 25 \\
& Com risco* $^{*}$ & 13 & 72,2 & 14 & 77,8 & 27 & 75 \\
\hline
\end{tabular}

* Classificação de acordo com os parâmetros da OMS, 1998. IMC: índice de massa corporal; CC: circunferência da cintura; n: número amostral.

O IMC médio da amostra observado foi de $27,9 \pm 5,9 \mathrm{~kg} /$ $\mathrm{m}^{2}$, sendo $27,7 \pm 5,9 \mathrm{~kg} / \mathrm{m}^{2}$ para as mulheres e de $28,1 \pm 6,0$ $\mathrm{kg} / \mathrm{m}^{2}$ para os homens. O estado nutricional de eutrofia representou $50 \%(\mathrm{n}=9)$ dos casos masculinos, seguido de $16,6 \%$ de sobrepeso $(n=3)$ e $33,3 \%$ de obesidade $(n=6)$. Para as mulheres, tanto o diagnóstico nutricional eutrófico, quanto o sobrepeso e a obesidade representaram $33,3 \%(n=6)$ cada. Dessa forma, 58,33\% $(\mathrm{n}=21)$ da amostra apresentaram sobrepeso ou obesidade.

Quanto ao risco representado pela medida elevada da circunferência da cintura (CC), a maioria dos homens (72,2\% $\mathrm{n}=13)$ e a maioria das mulheres $(77,8 \%, \mathrm{n}=14)$ apresentaram risco para desenvolvimento de doenças cardiovasculares segundo a classificação da $\mathrm{OMS}^{16}$. 
As informações relativas à avaliação antropométrica podem ser observadas na Tabela 2 .

Tabela 2: Comparação intergêneros dos valores antropométricos e da porcentagem de gordura corporal de pacientes atendidos no Ambulatório de Feridas do Campus Cedeteg, Unicentro, Guarapuava -PR.

\begin{tabular}{cccc}
\hline & Mulheres & Homens & Valor de p \\
\hline Massa corporal (kg) & $70,3 \pm 15,2$ & $83,8 \pm 19,3$ & $0,026^{*}$ \\
Estatura (m) & $1,59 \pm 0,06$ & $1,72 \pm 0,05$ & $0,001^{*}$ \\
IMC (kg/m $\mathbf{m}^{2}$ & $27,6 \pm 5,9$ & $28,0 \pm 5,9$ & 0,851 \\
$\mathbf{C C}(\mathbf{c m})$ & $92,0 \pm 11,6$ & $104,3 \pm 15,8$ & $0,012^{*}$ \\
GC (\%) & $37,7 \pm 4,8$ & $26,8 \pm 7,5$ & $0,001^{*}$ \\
CMB (cm) & $24,0 \pm 2,9$ & $26,0 \pm 3,6$ & 0,080 \\
\hline
\end{tabular}

*Teste de Mann-Whitney; $\mathrm{p}<0,05$. IMC: índice de massa corporal; $\mathrm{CC}$ : circunferência de cintura;\%GC: porcentagem de gordura corporal; CMB: circunferência muscular do braço; kg: quilogramas; m: metros; $\mathrm{kg}$ / $\mathrm{m}^{2}=$ quilogramas por metro quadrado; $\mathrm{cm}$ : centímetros.

Ao comparar as características antropométricas entre os gêneros observou-se para os homens valores significativamente superiores aos das mulheres para a massa corporal $83,8 \pm 19,3 \mathrm{~kg}(\mathrm{p}=0,026)$, para a estatura $1,72 \pm 0,05$ $\mathrm{m}(\mathrm{p}=0,001)$, para a CC $104,3 \pm 15,8 \mathrm{~cm}(\mathrm{p}=0,012)$ e para a CMB 26,0 $\pm 3,6 \mathrm{~cm}(\mathrm{p}=0,008)$. A porcentagem de gordura corporal $(\% \mathrm{GC})$ mostrou-se mais elevada nas mulheres $37,7 \pm 4,8 \%(p=0,001)$. As diferenças encontradas entre os gêneros já eram esperadas devido às particularidades fisiológicas.

Para ambos os grupos, homens e mulheres, respectivamente, a ingestão média de vitamina $A(p=0,001$ e $\mathrm{p}=0,002)$, vitamina $\mathrm{C}(\mathrm{p}=0,008$ e $\mathrm{p}=0,0016)$ e zinco $(p=0,080$ e 0,001$)$ mostrou-se abaixo do recomendado. No grupo das mulheres, o consumo calórico atual $(p=0,001)$, de ferro $(p=0,004)$ e proteínas $(p=0,020)$ também foi significativamente inferior às recomendações das DRIs ${ }^{24}$. Já no grupo dos homens, a ingestão de calorias $(p=0,267)$ apresentou-se adequada e a de proteínas $(p=0,879)$ foi estatisticamente superior. A ingestão de ferro $(p=0,007)$ apresentou-se acima do recomendado, porém sem ultrapassar os limites superiores toleráveis indicados pelo Institute of Medicine $^{24}$. Os valores do consumo e da recomendação dos nutrientes podem ser observados nas Tabelas 3 e 4 .

Tabela 3: Valores de ingestão e recomendação de nutrientes em homens atendidos no Ambulatório de Feridas do Campus Cedeteg, Unicentro, Guarapuava-PR.

\begin{tabular}{ccccc}
\hline \multirow{2}{*}{ Nutriente } & \multicolumn{4}{c}{ Homens } \\
\cline { 2 - 4 } & Ingestão & Recomendação** & Adequação (\%) & Valor de p \\
\hline Calorias (kcal) & $1994,1(974,3-2536,9)$ & $2033,1(1951,7-2400,4)$ & 98,1 & 0,267 \\
Proteínas (g) & $76,6(40,3-92,9)$ & $62,55(56,4-76,7)$ & 122,5 & 0,879 \\
Vitamina A (mcg) & $224,8(145,5-346,0)$ & 900,0 & 25,0 & $0,001^{*}$ \\
Vitamina C (mg) & $11,5(3,8-44,1)$ & 90,0 & 12,7 & $0,008^{*}$ \\
Zinco (mg) & $7,1(3,6-13,7)$ & 11,0 & 64,5 & 0,080 \\
Ferro (mg) & $12,8(6,6-19,3)$ & 8,0 & 160 & $0,007^{*}$ \\
\hline
\end{tabular}

*Teste pareado de Wilcoxon; $<<0,05$. Mediana (25 - 75); **Recomendação: Calorias: Equação de Harris Benedict (HBE) x 1,3 (fator atividade) x 1,0 fator injúria; Proteínas: $0,8 \mathrm{~g} / \mathrm{kg} / \mathrm{dia}$; Micronutrientes: DRIs segundo gênero e faixa etária; kcal: quilocalorias; g; gramas; mcg: microgramas; mg: miligramas.

Tabela 4: Valores de ingestão e recomendação de nutrientes em mulheres atendidas no Ambulatório de Feridas do Campus Cedeteg, Unicentro, Guarapuava -PR.

\begin{tabular}{ccccc}
\hline \multirow{2}{*}{ Nutriente } & \multicolumn{4}{c}{ Mulheres } \\
\cline { 2 - 5 } Ingestão & Recomendação** & Adequação (\%) & Valor de p \\
\hline Calorias (kcal) & $1188,2(1033,9-1364,2)$ & $1762,2(1612,6-1892,6)$ & 67,4 & $0,001^{*}$ \\
Proteínas (g) & $43,9(35,5-58,2)$ & $58,6(46,3-66,4)$ & 74,9 & $0,020^{*}$ \\
Vitamina A (mcg) & $280,7(146-572,6)$ & 700,0 & 40,0 & $0,002^{*}$ \\
Vitamina C (mg) & $10,6(5,4-80,9)$ & 75,0 & 14,1 & $0,016^{*}$ \\
Zinco (mg) & $4,0(1,7-6,1)$ & 11,0 & 36,4 & $0,001^{*}$ \\
Ferro (mg) & $7,1(5,1-8,9)$ & 8,0 e $18,0 * * *$ & 88,7 e 39,4 & $0,004^{*}$ \\
\hline
\end{tabular}

*Teste pareado de Wilcoxon; $<<0,05$. Mediana (25 - 75); **Recomendação: Calorias: Equação de Harris Benedict (HBE) x 1,3 (fator atividade) x 1,0 fator injúria; Proteínas: $0,8 \mathrm{~g} / \mathrm{kg} / \mathrm{dia}$; Micronutrientes: DRIs segundo gênero e faixa etária. *** Algumas mulheres tinham necessidades diferentes devido à faixa etária. kcal: quilocalorias; g; gramas; mcg: microgramas; mg: miligramas.

As úlceras de perna passaram a ser vistas como problema de saúde pública devido ao aumento da incidência na população, principalmente de idosos de baixa renda por se tratar de uma enfermidade que pode demorar longos períodos 
para cicatrizar $^{25,26}$.

Comorbidades, como o diabetes melittus ou hipertensão arterial sistêmica, principalmente quando não tratadas, podem contribuir para o agravamento, dificuldade de cicatrização e recidivas das úlceras ${ }^{27}$.

Nesse trabalho, encontrou-se uma faixa etária que variou de 40 a 76 anos, sendo a maioria adulta (52,8\%). Pesquisa semelhante apresentou uma variação de idade de 27 a 94 anos, com uma maioria de idosos $(58,9 \%)^{1}$. De acordo com Yamada et al. ${ }^{28}$, estudos brasileiros apontam maior incidência de úlceras venosas entre as mulheres e idosos, especialmente acima de 65 a 70 anos de idade.

$\mathrm{Na}$ presente pesquisa, o tipo prevalente de úlcera foi o venoso com $72 \%$, seguido de neuropática por hanseníase com $20 \%$ e outros tipos com $8 \%$, resultado que corrobora com os achados de Frade et al. ${ }^{1}$, que também encontraram maior prevalência de úlceras do tipo venoso (79\%), seguido pelas hipertensivas $(15,3 \%)$, as mistas $(4,8 \%)$ e neoplásicas ou hansênicas $(0,9 \%)$. Do mesmo modo, não havia nenhum caso de úlcera por diabetes isolada.

Segundo Borges $^{29}, 70 \%$ a $90 \%$ dos casos de úlceras de perna são representados pela úlcera de estase venosa. Além disso, a mesma é considerada problema de saúde pública mundial e em nosso país já é a $14^{\mathrm{a}}$ causa de afastamento provisório das atividades laborais e $32^{\mathrm{a}}$ razão de afastamento definitivo do trabalho ${ }^{30}$, justificando a importância da prevenção e tratamento das doenças associadas.

Vinte por cento da amostra apresentou úlceras do tipo neurotrófica por sequelas da hanseníase. Indicadores epidemiológicos sugerem que, no município de Guarapuava$\mathrm{PR}$, a endemia de hanseníase ainda é elevada. Este fato pode explicar o grande número de úlceras desse tipo na amostra ${ }^{31}$.

Nos serviços de saúde da rede básica do país existe uma grande incidência de úlceras localizadas na planta dos pés devido à perda da sensibilidade e traumas. Entretanto, o surgimento também pode estar relacionado à paralisia dos músculos do pé, endurecimento das articulações e pele anidrótica $^{11}$.

No estudo em questão, pode-se observar uma prevalência maior de $\operatorname{HAS}(52,8 \%, \mathrm{n}=19)$ dentre as comorbidades presentes. Guterres ${ }^{32}$, em seu estudo com portadores de úlcera de perna com idade entre 28 e 81 anos, também encontrou a hipertensão arterial como doença mais prevalente em 73,8\% dos casos $(n=14)$, porém a segunda maior comorbidade foi o diabetes $(34,8 \%, n=8)$, diferentemente do que foi observado no presente trabalho, no qual a obesidade $(33,3 \%, \mathrm{n}=12)$ e a insuficiência venosa crônica $(30,6 \%, n=11)$ foram mais prevalentes que os casos de diabetes $(19,4 \%, n=7)$.

Cerca de 58\% da amostra apresentou o diagnóstico nutricional de sobrepeso ou obesidade de acordo com o IMC. Segundo Wipke-Tevis e Stotts ${ }^{33}$, em estudo com 32 pacientes portadores de úlceras venosas, $60 \%$ dos avaliados também apresentou as condições de sobrepeso ou obesidade.

Desse modo, o tratamento de feridas crônicas deve estar voltado para a condição subjacente e comorbidades, tais como diabetes mellitus, hipertensão arterial sistêmica, insuficiência venosa crônica, obesidade ou desnutrição, a fim de planejar adequadamente a assistência ${ }^{13,34}$.

A relevância da pesquisa sobre o estado nutricional e prevalência de doenças crônicas em pacientes com úlceras de perna está no fato dessa população apresentar hábitos alimentares inadequados que dificultam a cicatrização, seja pela falta de nutrientes ou pelo excesso desses ${ }^{27}$.

As vitaminas são co-fatores importantes de várias enzimas no processo de cicatrização. A vitamina A é necessária para a manutenção da epiderme normal e para a síntese de glicoproteínas e proteoglicanas, sua deficiência retarda a reepitelização das feridas e a síntese de colágeno. Já a vitamina $\mathrm{C}$ atua na cicatrização de feridas através da hidroxilação dos aminoácidos lisina e prolina, os quais são essenciais na formação do colágeno e na proliferação dos fibroblastos ${ }^{14}$.

Nesse sentido, verificou-se que o consumo alimentar habitual dos indivíduos avaliados nessa pesquisa estava inadequado para todos os micronutrientes em ambos os gêneros, o que pode estar relacionado aos seus hábitos de baixa ingestão de frutas e hortaliças, alimentos fontes desses nutrientes.

Adicionalmente, a ingestão calórica, de ferro e de proteína estava insuficiente somente no grupo das mulheres. Esses dados corroboram com outro estudo de úlceras venosas que apontou consumo calórico insuficiente em $85 \%$ da amostra, baixa ingestão de proteína em $75 \%$ e de zinco em $85 \%$, revelando ingestão inadequada para satisfazer as necessidades para a cicatrização e cura das lesões ${ }^{33}$.

Tobón et al. $^{6}$ estudaram alimentação de 8 pacientes com úlceras venosas e perceberam consumo abaixo do recomendado para a proteína em um paciente (12,5\%), vitamina $\mathrm{C}$ em quatro pacientes $(50 \%)$ e de zinco em três pacientes $(37,5 \%)$.

É confirmado pela literatura científica que a carência de zinco e selênio retardam a cicatrização de feridas, pois, essas deficiências afetam todas as fases da reparação tecidual. A anemia também pode contribuir para a diminuição da formação de colágeno e aumentar a susceptibilidade do tecido ao desenvolvimento de úlceras e outros tipos de lesões ${ }^{14}$.

Assim como ocorreu no presente estudo, no trabalho de Teixeira et al. ${ }^{35}$ com pacientes portadores de úlcera de pressão foi verificado que a ingestão de proteínas, energia e micronutrientes (vitaminas A, C e E, cobre e zinco) foram insuficientes. Os mesmos autores concluíram que ainda faltam estudos que comprovem uma eficácia da suplementação de nutrientes em pacientes com úlceras, porém todos concordam que uma melhora no estado nutricional favorece a cura e prevenção de úlceras na presença de outros fatores associados.

\section{Conclusão}

Os resultados desse estudo apontaram grande prevalência de doenças crônicas secundárias, como hipertensão arterial 
sistêmica, diabetes e obesidade. Ainda, observaram-se hábitos alimentares inadequados que desfavorecem a cicatrização das úlceras.

Juntamente com os cuidados com a ferida, há a necessidade de incentivar uma alimentação equilibrada que beneficie a cicatrização e atue sobre os fatores que predispõem o desenvolvimento e dificultam a cicatrização das úlceras. Pois, além de reduzir o tempo de tratamento e o custo por ele gerado, a terapêutica nutricional pode contribuir para amenizar o sofrimento do paciente.

Diante do exposto, fica evidente a importância de incluir o nutricionista na equipe multiprofissional para o tratamento de pacientes com úlceras crônicas de membros inferiores.

\section{Referências}

1. Frade MAC, Cursi IB, Andrade FF, Soares SC, Ribeiro WS, Santos SV, et al. Úlcera de perna: um estudo de casos em Juiz de Fora-MG (Brasil) e região. An Bras Dermatol 2005;80(1):41-6.

2. Brown A. Does social support impact on venous ulcer healing or recurrence? Br J Community Nurs 2008;13(3):6-10

3. Werchek S. Diagnosis and treatment of venous leg ulcers. Nurse Pract 2010;35(12):46-53.

4. Abbade LPF, Lastóia S, Rollo HA, Stolf HO. A sociodemographic, clinical study of patients with venous ulcer. Int J Dermatol 2005;44:449-56.

5. Reichenberg J, Davis M. Venous ulcers. Semin Cutan Med Surg 2005;24:216-26.

6. Tobón J, Whitney JD, Jarret M. Nutritional status and wound severity of overweight and obese patients with venous leg: a pilot study. J Vasc Nurs 2008;26(2):43-52.

7. Browse NL, Burnand KG, Irvine AT, Wilson NM. Úlcera venosa: diagnóstico. In: Browse NL, Burnand $\mathrm{KG}$, Irvine AT, Wilson NM. Doenças venosas. Rio de Janeiro: Di-livros; 2001. p.485-520.

8. Valencia IC, Falabella A, Kirsner RS, Eaglstein WH. Chronic venous insufficiency and venous leg ulceration. $J$ Am Acad Dermatol 2001;44(3):401-21.

9. Smeltzer SC, Bare BG. Brunner \& Suddarth: históricos e cuidados aos pacientes com distúrbios vasculares e problemas da circulação periférica. In: Smeltzer SC, Bare BG. Tratado de enfermagem médico-clinica. Rio de Janeiro: Guanabara Koogan; 2009. p.823-74.

10. Vowden P. Leg ulcers: assessment and management. Indep Nurse 2010(1):30-3.

11. Brasil. Ministério da Saúde, Secretaria de Políticas de Saúde, Departamento de Atenção Básica. Manual de condutas para úlceras neurotróficas e traumáticas / Ministério da Saúde, Secretaria de Políticas de Saúde, Departamento de Atenção Básica. Brasília: Ministério da Saúde; 2002.

12. Irion G. Insuficiência vascular. In: Irion G. Feridas: novas abordagens, manejo clínico a atlas em cores. Rio de Janeiro: Guanabara Koogan; 2005. p.145-60.

13. Poletti NAA. O cuidado de enfermagem a pacientes com feridas crônicas. A busca de evidências para a prática. Dissertação [Mestrado em Enfermagem] - Universidade de São Paulo; 2000.

14. Bottoni A, Bottoni A, Rodrigues RC, Celano RMG. Papel da nutrição na cicatrização. Rev Ciênc Saúde 2011;1(1):98-103.
15. Sistema de Vigilância Alimentar e Nutricional. Vigilância alimentar e nutricional - SISVAN: orientação básica para a coleta, o processamento, a análise de dados e a informação em serviços de saúde. Brasília: Ministério da Saúde; 2004.

16. World Health Organization. Obesity: preventing and managing the global epidemic. Geneva: WHO; 1998.

17. Organização Pan-Americana da Saúde-OPAS. Informe preliminar da $36^{\mathrm{a}}$ Reunión del Comité Asesor de Investigaciones em Salud - Encuesta Multicéntrica. Salud Bienestar y Envejecimento (SABE) em América Latina y el Caribe; 2002.

18. Chumlea WC, Roche AF, Steinbaugh ML. Estimating stature from knee height for persons 60 to 90 years of age. J Am Geriatr Soc 1985;33:116-20.

19. Pollock ML, Wilmore JH. Exercícios na saúde e na doença: avaliação e prescrição para prevenção e reabilitação. Rio de Janeiro: Médica Científica; 1993.

20. Harris JA, Benedict FG. Biometric studies of basal metabolism in man. Washington: Carnegie Institute of Washington; 1919.

21. Long CL, Schaffel BS, Geiger JW, Schiller WR, Blakemore WS. Metabolic response to injury and illness: estimation of energy and protein needs from indirect calorimetry and nitrogen balance. J Parenter Enteral Nutr 1979;3:452-6.

22. Institute of Medicine, Dietary reference intakes for Energy, Carbohydrates, Fiber, Fat, Fatty Acids, Cholesterol, Protein and Amino Acids (Macronutrients). Washington: National Academy; 2000.

23. Institute of Medicine. Dietary reference intakes for vitamin C, vitamin E, selenium, and carotenoids. Washington: National Academy; 2000.

24. Institute of Medicine. Dietary reference intakes for vitamin A, vitamin $\mathrm{K}$, arsenic, boron, chromium, copper, iodine, iron, manganese, molybdenum, nickel, silicon, vanadium, and zinc. Washington: National Academy; 2002.

25. Silva FAA, Freitas CHA, Jorge MSB, Moreira TMM, Alcântara MCM. Enfermagem em estomaterapia: cuidados clínicos ao portador de úlcera venosa. Rev Bras Enferm 2009;62(6):889-93.

26. Benevides JP, Coutinho JFV, Santos MCL, Oliveira MJA, Vasconcelos FF. Avaliação clínica de úlceras de perna em idosos. Rev Rene 2012;13(2):300-8.

27. Tenório, EB; Braz MA. Intervenção do enfermeiro como diferencial de qualidade no tratamento de feridas. Rev Bras Home Care 2002;10(2):4.

28. Yamada BFA. Úlceras Venosas. In: Jorge AS, Dantas SRPE. Abordagem Multiprofissional do tratamento de Feridas. São Paulo: Atheneu; 2003. p.247-59.

29. Borges EL. Tratamento tópico de úlcera venosa: proposta de uma diretriz baseada em evidências. [Doutorado em Enfermagem] - Escola de Enfermagem, Universidade de São Paulo; 2005.

30. Nunes PJ. Avaliação da assistência à saúde dos portadores de úlceras venosas atendidas no Programa Saúde da Família do Município de Natal/RN. Dissertação. [Mestrado em Enfermagem] - Universidade Federal do Rio Grande do Norte; 2006.

31. Paraná. Secretaria de Estado da Saúde do Paraná, Departamento de Vigilância e Controle em agravos estratégicos. Programa de Controle da Hanseníase. Taxa de detecção Geral e Menor de 15 anos e Taxa de Prevalência por Regional de Saúde e Municípios -2007. 2008. Curitiba: SES; 2008 
32. Guterres LB, Bordin R. Qualidade de vida dos portadores de úlcera em perna cadastrados nas unidades de saúde do município de Cachoeirinha-RS. Porto Alegre: Universidade Federal do Rio Grande do Sul; 2010.

33. Wipke-Tevis DD, Stotts NA. Nutrition, tissue oxygenation and healing of venous leg ulcers. J Vasc Nurs 1998;16:48-56.

34. Tatsioni A, Balk E, O'Donnell T, Lau J. Usual care in the management of chronic wounds: a review of the recent literature. J Am Coll Surg 2007;205(4):617-24.

35. Teixeira ES, Pinto JD, Araújo CG, Baptista DR, Lopes JP. Avaliação do estado nutricional e do consumo alimentar de pacientes amputados e com úlceras de pressão atendidos em um Centro Hospitalar de Reabilitação. Saúde Mundo 2011;35(4):448-53. 